\title{
A INSULINA PROTAMINA ZINCO NO TRATAMENTO DO DIABETES (*)
}

\author{
DR. CARLOS MACEDO RIBEIRO \\ Assistente de Clin. Propedeutica Medica (Prof. Jairo Ramos) da Escola \\ Paulista de Medicina
}

O tratamento do diabetes pela insulina comum (I. C.), apresenta alguns inconvenientes, na maior parte decorrentes da rapidez de ação, que se afasta do ritmo fisiológico do organismo.

Assim, a ação hipoglicemiante cuja duração é, no maximo, de oito horas obriga-nos a fazer, nos casos graves, duas, tres e mesmo quatro injeções por dia.

Em certos pacientes, manifesta-se uma onda hiperglicemica de madrugada que Joslin tentava remediar com a injeção de uma pequena dose de insulina ás dez ou onze horas da noite, portanto, mais uma injeção. No diabetes infantil e' no do adulto deste tipo, a grande labilidade da glicemia faz com que se observem grandes quedas da mesma após a' injeção de insulina, com desagradaveis fenômenos hipoglicemicos e apezar disso, o doente pode apresentar glicosúria no intervalo das injeções.

Para remediar esses inconvenientes, porfiaram os pesquizadores por obiter uma insulina de ação mais prolongada. Estas tentativas já vêm de pouco após a descoberta da insulina por Banting e Best, em 1922. 'Primeiramente ensaiaram' tornal-a insoluvel, misturando-a com certas substancias como a goma arabica (Burgess, Campbell e outros, 1923), a gelatina, a lecitina, o tanino, os ferricianetos. Tentaram colocal-a em suspensão oleosa para retardar a absorção como fizeram Hedvall em 1925 e Leyton em 1929.

Em todas essas tentativas obteve-se muitas vezes uma ação retardada e mais ou meno satisfatória nos casos levés; mas quando se necessitavam doses mais elevadas, a ação se evidenciava bastante irregular, ocorrendo fortes reações hipoglicemicas.

Clausen, em 1935, adicionou pequenas doses de adrenalina para refreàr a queda rapida da glicemia. Brunnengraber adiciona extrato de lobo posterior da hipófise, com bons resultados (Deposulin). A Surfen-insulin (com um antisséptico) tem a desvantagem de provocar reações locais.

(*) Trabalho apresentado na Associaçāo Paulista de Medicina. 
Hagedorn, cientista dinamarquês, orientou suas investigações noutro sentido: tentou obter e conseguiu, uma insulina quasi insoluvel nos meios orgânicos, formando um composto de insulina com uma protamina retirada do esperma do salmão (Salmo iridius) cuja menor solubilidade se encontra num $\mathrm{pH}$ igual a 7,3 ou seja mais ou menos o mesmo do tecido animal onde se faz a injeção. Injetada essa solução, liberta-se a insulina regular e lentamente, prolongando-se sua ação por doze ou dezeseis horas (o dobro da ação da ins. comum).

Depois de dois anos de experiências, apresentou esses trabalhos no Congresso Nórdico de Medicina Interna, reunido em Copenhagen em Junho de 1935. Em 18 de Janeiro de 1936, publica no Journal of the Am Med. Association um artigo sobre a nova insulina. Nesse mesmo numero varios colaboradores de Joslin publicam tambem um trabalho confirmando as conclusões de Hagedorn.

Nesse tempo, era necessario fazer a mistura no momento de usar, da insulina comum com, a solução contendo a protamina com a substancia tampão (fosfato de sodio). Essa mistura durava no máximo algumas semanas, o que era um inconveniente grande.

Em princípios de 1937, apareceu no mercado a insulina protamina zinco (I. P. Z.) que substituiu completamente a insulina protamina pois além de se apresentar já misturada, tem ação mais protraida e muito maior durabilidade.

A adição do zinco, decorreu dos belos trabalhos de Scott e Fisher sobre a ação deste metal no reforço da ação hipoglicemiante da insulina protamina e no retardamento de sua absorção.

A I. P. Z. apresenta-se a venda sob a forma dè um liquido de côr leitosa correspondendo a 40 unidades de insulina e $0,08 \mathrm{mg}$. de zinco por centimetro cubico. Quando em repouso, apresenta um deposito esbranquiçado e porisso, antes de usar, o vidro deve ser agitado brandamente de modo a se obter uma suspensão homogênea, devendo a injeção ser feita no tecido celular subcutaneo (1).

\section{VANTAGENS DA INSULINA PROTAMINA ZINCO}

Sua ação prolongada permite reduzir as doses a uma só, diária. A ação prolonga-se por mais de 24 horas (3-4 vêzes a da ins. comum), conforme a dose.

Não se observam grandes oscilações da glicemia, evitando-se seja a hipoglicemia posinsulínica como as hiperglicemias tardias, particularmente a noturna. Essa ação regular evidencia-se bem no gráfico abaixo, em que se nota que 30 u. de I. P. Z. mantêm a glicemia dum diabético dentro dos limites normais, fato que não se observa com 36 u. de ins. comum no mesmo paciente. (1) A insulina utilizada neste trabalho foi a da fabrica americana Eli Lilly and
Company. (Protamine-zinc-insulin). 


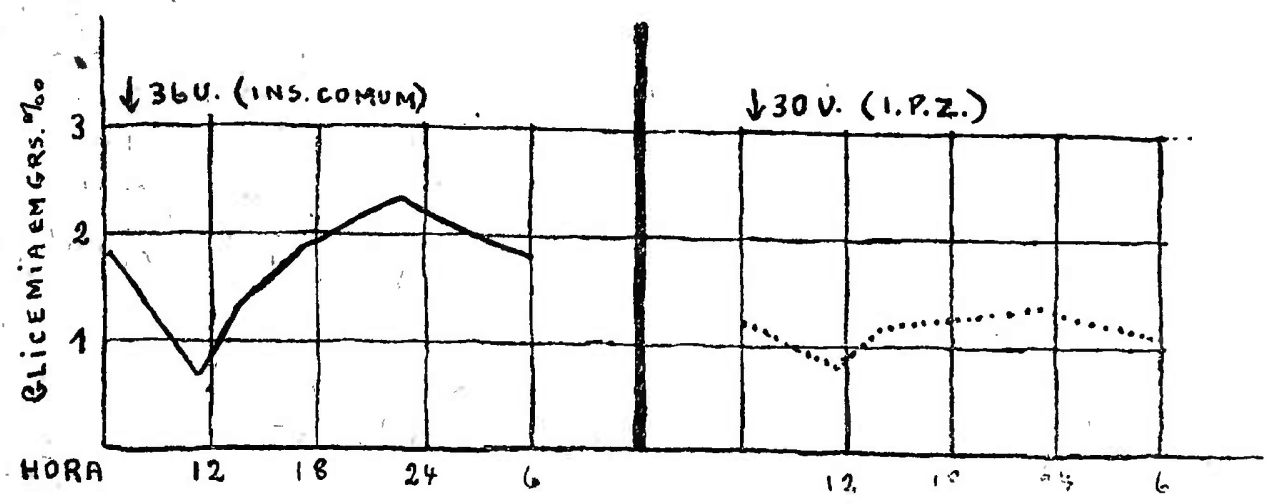

MODO DE USAR E DIFICULDADES

Em pacientes que iniciam o tratamento, segundo a norma de Joslin, principiar logo com a I. P. Z. e si o paciente tem cetonúria, adicionar a ins. comum, principalmente nos primeiros dias. $\mathrm{O}$ aumento da dose de I. P. Z. deve ser feito gradativamente, de 4 a 10 u., sempre guardando um intervalo de 3 dias no minimo, pois devido á ação cumulativa da I. P. Z. seus efeitos se patenteiam bem só após 3 dias.

Com as dietas de teor moderado em hidratos de carbono (150 a 180 grs.) obtem-se em maior número de casos o contrôle com uma só injeção, emquanto que nas dietas com 180 a 300 grs. de $H$. de C. é frequęnte a necessidade de uma ou duas injecções adicionais de ins. comum.

Nestes casos é preferivel dar uma dose basal moderada de I. P. Z. e controlar a glicosúria com a ins. comum. A vantagem da I. P. Z. reside no fato de refrear a onda hiperglicemica noturna e manter a glicemia diária dentro de limites razoáveis.

A I. P. Z. tem sido usada com sucesso no tratamento do diabetes em geral, nos casos de diabetes com tuberculose, gangrena, antrás, casos cirúrgicos, e mesmo na acidose e coma.

No diabetes infantil, os resultados têm sido ótimos como se pode vêr nas estatísticas de P. White (da clínica de Joslin), em que os bons resultados somam 94 a 96 por cento dos casos.

Na clínica de Joslin verificou-se que a I. P. 'Z. tem uma ação muito favoravel na hepatomegalia das crianças diabéticas, pois essa hepatomegalia, tão frequente, regride de modo notavel com o uso da I. P. Z.

Um fato de grande importância que precisa ser bem conhecido dos médicos e pacientes que usam a I. P. Z., é a modalidade diferente da exteriorisação sintomática da reação hipoglicemica.

As queixas principais dos pacientes são: cefaléa (sintoma muito frequente), náuseas, depressão, amnésia, não sendo tão frequentes os suores, tremores e sensação de fome tão conhecidos na reação hipoglicemica com a ins. comum. Não é raro encontrar-se uma glicemia de $0,40-0,50$ completamente asintomática. Estas cifras baixas são 
mais frequentes no periodo da madrugada ( 3 a 7 da manhã), como mostraram Neuhoff e Rabinovitch. .

O médico que usa a I. P. Z., deve conhecer a possibilidade de sobrevir um cọma hipoglicemico sem fenômenos premonitórios.

Alguns casos de hipoglicemia mortal foram já publicados, e experimentalmente, Hédon e outros, em cães, demonstraram a occorrencia de alterações nervosas não reversiveis, como consequência da hipoglicemia prolongada.

$\mathrm{Na}$ prática, quando a dose excede 40 unidades, é necessário ficar de sobreaviso e principalménte não exceder essa dose si o paciente não estiver sob contrôle médico frequente.

Si o paciente é idoso, com alterações das coronárias, é preferivel tolerar uma pequena glicosúria, antes que submetel-o ao risco de uma reação hipoglicemica que irá lesar o miocárdio, muitas vezes de modo permanente.

\section{RESULTADÓS PESSOAIS}

Em 30 casos de clínica particular e acompanhados por mais de um ano, sendo o paciente mais idoso de 73 anos e o mais jovem de $51 / 2$ anos, obtive o seguinte resultado:

$$
\begin{gathered}
\text { Em uso da I.P.Z. } \\
" \text { (sòmente) } \ldots \ldots \ldots \ldots \ldots
\end{gathered}
$$

Dos 22 casos còm I. P. $Z: 17$ ou $77,2 \%$ necessitaram menos que 30 u. e 5 ou $22,8 \%$, entre 30 e 50 u.

As dietas oscilaram entre 180 e 260 gr's. de H. de C., proteinas entre 0,8 e $1 \mathrm{gr}$. por kilo, e gorduras entre 50 e 90 grs.

$\mathrm{Na}$ maioria dos casos a injeção de I. P. Z. era feita ás 8 da manhã e em alguns casos ás 11 horas, antes do almoço. Em grande numero de casos a I. P. Z. era adicionada á I. comum na mesma seringa segundo a técnica recomendada por Lawrence (aspirar primeiro a I. comum).

A dose maxima de İ. P. Z. foi 100 u., de manhã, numa paciente de tipo acromegálico, sem alteração radiológica da hipófise e que não se benreficiou da irradiação dessa glândula.

Noutro caso, foi levada a termo uma gravidez com o uso da $I$. P. Z. (em media 50 u.), mais 20 a 30 u. da ins. comum. A paciente tivera 3 abortos desde que se iniciou o diabetes. Infelizmente, nossos esforços não impediram que a paciente tivesse um hidramnios e um natimorto. Nesse caso havia um fatộ "diabetes insipidus" pois a paciente urinava 3 a 4 litros, mesmo aglicosúrica.

Uma paciente hipertensa que sofria crises de angor e necessitava 3 injeções de ins. comum por dia, passou a usar a I. P. Z. mais uma injeção de ins. comum, sem apresentar acidentes. Neste caso sempre foi tolerada uma ligeira glicosúria para evitar o risco de uma hipoglicemia. 
Numa criança de 5 1/2 anos que necessitava de 2 injeções de ims. comum, estabeleceu-se um equilibrio satisfatório e aumento de peso, com o uso de uma só injeç̧ão de I. P. Z. (18 u.), com uma dieta de 180 grs. de $\mathrm{H}$. de $\mathrm{C}$. sociada:

São os seguintes os resultados que obtive com I. P. Z. só ou as-

$$
\begin{aligned}
& \text { Excelente (aglicosúrico) …........... } 9 \text { casos }(30 \%) \\
& \text { Bom (glicosúria menor que } 10 \mathrm{p} / \text { litro }) \ldots 15 \text {. } \ldots(50 \%) \\
& \text { Regular (" maior " } "(") \text { ) } 6 \text {. } 6 \text { (20\%) }
\end{aligned}
$$

Os 6 casos de resultado regular, referem-se a pacientes que não observavam exatamente o regime, e, portanto, não devem ser levados à conta de um fracasso do medicamento.

Concluindo, podemos dizer que a I. P. Z. representa um grande progress̀o no tratamento do diabetes, pois com uma só injeção podemos controlar perfeitamente os casos leves e muitos de media gravidade. Nos restantes, casos medios e graves, a adição judiciosa da I. comum à I. P. Z. resolve satisfatóriamente a maioria dos casos.

Um fato constatado por varios autores, e que tambem notei, é que os pacientes embora com glicosúria, sentem-se melhor com a I. P. Z. que com a I. comum.

\section{RESUMO}

O A. tratando da Insulina protamina zinco, faz um pequeno histórico, relata suas vantagens, indicações e resultados. Refere os resultados pessoais obtidos em 30 casos acompanhados por mais de um ano, em que obteve bons resultados em $80 \%$ dos casos, seja com a I. P. Z. isoladamente, seja associada a uma injeção de insulina comum. Os $20 \%$ restantes não podem ser apontados. como fracasso da I. P. Z. desde que nesses casos a inobservância do regime por parte dos pacientes, impediu que se tirasse uma conclusão verdadeira.

\section{SUMMARY}

Protámine zinc insulin, represents a distinct advance in diabetic treatment. Almost all of my patients now under treatment who require insulin are receiving protamine zinc insulin (Lilly). This paper reports the results of treatment of 30 patients with a follow up of a year, with prot. zinc. ins. either used alone or combined with a supplementary dose of unmodified insulin. The results were in $30 \%$ excellent, in $50 \%$ good and in $20 \%$ poor (uncooperative patients). The diet ranged between 180 and 260 grams of carbohydrates; $0,8-$ 1 gram of protein per kilo of body weight and fat less than 100 grams. 


\section{BIBLIOGRAFIA (resumida)}

1 - H. G. Hagedorn; Norman Jensen; N. B. Kraurup; I. Wodstrup: Protamine Insulinate, J.A.M.A. 106: 177 (January 18) 1936.

2 - Howard. F. Root; Priscilla White; Alexander Marble and E. H. Sтотz: Clinical experience with protamine insulinate, J.A.M.A. 106: 180 (Jan. 18) 1936.

3 - Herman O. Mosenthal: Protamine Zinc Insuline; J.A.M.A. 110: 87 (Jan. 8) 1938.

4 - Elliot P. Joslin: Difficulties in the use of Protamine Zinc Insulin, J.A.M.A. 110 : 90 (Jan. 8) 1938.

5 - Edwin J. KePLER: Clinical experience with Protamine Zinc Insulin, J.A.M.A. $110: 92$ (Jan. 8) 1938.

6 - Priscilla White and Lovilla Winterbottom: Treatment of diabetic girls, J.A.M.A. 112 : 1440 (April 15) 1939.

7 - Herman O. Mosenthal: The prolonged use of Protamine Zinc Insulin, J.A.M.A. $113: 17$ (July 1) 1939.

8 - NeuhofF and Rabinovitch: Protamine Zinc Insulin. Clinical Observations and comparative analysis of blood sugar curves obtained with use of Protamine Zinc Insulin and with regular insulin, Arch. Int Med. 62 : 447 (Sept.) 1938.

9 - R. Boulin, M. UllmanN, R. Mallet et H. Bour: L'insuline-protaminezinc dans le traitement du diabète sucré: Annales d'Endocrinologie, Tome 3.n.o 3, pg. 270, 1939.

10 - R. D. LAWRENCE: Z.P.I. in diabetes treatment by one daily injection, Br. M. J. 1 : 1077 (May 27) 1939.

11 - Heinsen und Reinwein: Über die Behandlung von Zuckerkranken mit P.Z.I. Deutsche Med. Wchnschr. $64: 325: 1938$.

12 - Boller und Pilgerstorfer: Die Hypoglykämie bei Pr. Z. Insulinanwendung. Klin. Wchnschr. $17: 1065: 1938$.

13 - F. UMber, F. K. StöRRING und E. GLET: Klinische und ambulante Erfahrungen mit verschiedenen Insulindepotpräparaten an 250 Diabetikern. Klin. Wchnschr. $17: 190: 1938$.

14 - P.' Gotтlebe: Klinische Erfahrungen mit Depotinsulimen. Klin. Wchnschr. $18: 485: 1939$.

15 - KERR and BEST: The effects of protamine insulin and related compounds in normal and depacreatized dogs: Am. J. M. Sc. 194: 149 (August) 1937

16 - L. Hédon, A. Loubatières et G. Heymann: Etude des effets de l'insuline-protamine zinc chez le chien dépancréaté: Annales d'Endocrinologie. Tome $1:$ n. 1 : pg. $97: 1939$.

17 - Carlos A. Campos: El tratamiento actual de la diabetes.. B. Aires, 1940. 